\title{
Fast Short Circuit Type I Detection Method based on $V_{\mathrm{GE}-M o n i t o r i n g}$
}

\author{
C. Herrmann, X. Liu, J. Lutz and T. Basler, \\ Chair of Power Electronics \\ Chemnitz University of Technology \\ Chemnitz, Germany
}

\begin{abstract}
In this paper, a Short Circuit type I detection method based on the monitoring of the gate voltage is investigated. The proposed detection principle relies on an existing method, which was realized as an integrated solution before. A modified discrete circuit solution is introduced, developed and tested. Moreover, measurements and investigations on different packaging concepts and test conditions are performed. The overview of the functionality, reliability, and restraints of this method, as well as aspects of a supposed dynamic self-adaption feature, are discussed.
\end{abstract}

Keywords: IGBT, Short Circuit Detection, Gate Drive Unit

\section{INTRODUCTION}

A low inductive Short Circuit type I (SC I) event, also known as Hard Switching Fault (HSF), appears when a power transistor is turned on to an already shorted load. It is a critical situation that should be detected and turned off as fast as possible, especially for transistors with reduced short circuit capability, such as on-stateoptimized IGBTs [1] [2].

The failure detection based on the desaturation of the power transistor is a well-known and reliable solution but lacks a fast detection time due to the necessity of a blanking time. It also requires space for the circuitry because of the use of high voltage blocking diodes or ohmic-capacitive dividers [3].

Several alternative approaches based on the monitoring of different quantities were proposed. They either interpret one quantity solely, e.g. the load current slope $\mathrm{d} i / \mathrm{d} t$ [4], the Gate-Emitter voltage $V_{\mathrm{GE}}$ [5] [6], or more than one quantity in combination. A 2-D detection presented and described in [1] [2] [7] is based on the simultaneous monitoring of both the load-current transient $\mathrm{d} i_{\mathrm{C}} / \mathrm{d} t$ and the gate voltage $V_{\mathrm{GE}}$. In addition, a detection combining a $V_{\mathrm{GE}}$ and $V_{\mathrm{CE}}$ evaluation was published in [8].

This paper provides an overview of an SC I detection method which is based solely on the monitoring of the gate voltage of the transistor. The SC detection principle was first introduced in [5], where it was realized via an integrated circuit. In this paper, a modified discrete circuit solution is developed and tested. Moreover, investigations on different packaging concepts and test conditions are shown, to provide a more complete overview of the functionality, reliability, restraints, and features of this method.

\section{FUNCTIONAL PRINCIPLE}

The detection circuit, which monitors the course of the Gate-Emitter voltage $V_{\mathrm{GE}}$ during the turn-on transition of the IGBT, is depicted in the bottom part of Fig. 1. For a normal turn-on process, the Miller-plateau can be observed in the $V_{\mathrm{GE}}$ course caused by the decreased Collector-Emitter voltage $V_{\mathrm{CE}}$, which will not take place due to the permanently high $V_{\mathrm{CE}}$ voltage under low inductive SC type I conditions. This effect was already discovered and used for detection schemes in [9] and [10] However, for a high inductive SC type I, a Miller-plateau would be observed due to the transient decrease of $V_{\mathrm{CE}}$. Therefore, the proposed method would not work reliably for the latter case.

$V_{1}$ and $V_{2}$ describe the voltage level of the capacitors $C_{1}$ and $C_{2}$, respectively. When the IGBT receives the turnon signal, $C_{1}$ and $C_{2}$ are individually charged by the currents $I_{1}$ and $I_{2} . I_{1}$ is adjusted higher than $I_{2}$, which results in a higher charging speed of $V_{1}$ for $C_{1} \approx C_{2}$. Two reference levels $V_{\text {Ref1 }}$ and $V_{\text {Ref2 }}$ are defined to stop the charging process of $V_{1}$ and $V_{2}$, respectively. $V_{\text {Ref1 }}$ is set below the Miller-plateau level $V_{\text {Miller. }}$ Meanwhile, $V_{\operatorname{Ref} 2}$ is fixed slightly lower than the steady-state value of the positive gate voltage. $V_{1}$ stops rising at time $t_{1}$ when $V_{\mathrm{GE}}$ reaches the value of $V_{\text {Ref1 }}$. Similarly, $V_{2}$ stops to increase at $t_{2}$, when $V_{\mathrm{GE}}$ reaches the value of $V_{\mathrm{Ref2}}$. The values of $V_{\mathrm{GE}}$ and the reference voltages $V_{\mathrm{Ref} 1}$ and $V_{\mathrm{Ref} 2}$ are halved to become processible by the comparators. For $V_{\mathrm{GE}}$ this is enabled via an ohmic voltage divider. $V_{1}$ and $V_{2}$ can be held across $C_{1}$ and $C_{2}$ by employing two Schottky diodes D1 and D2. At $t_{2}, V_{1}$ and $V_{2}$ are compared by Comp3.

The working principle is depicted in the schematic drawings in Fig. 2 and Fig. 3 for a faultless and an SC I turn-on process under consideration of idealistic courses 
for $V_{1}, V_{2}$, and the Fault-Signal $V_{\text {Fault }}$ as well as typical courses of $V_{\mathrm{GE}}$.

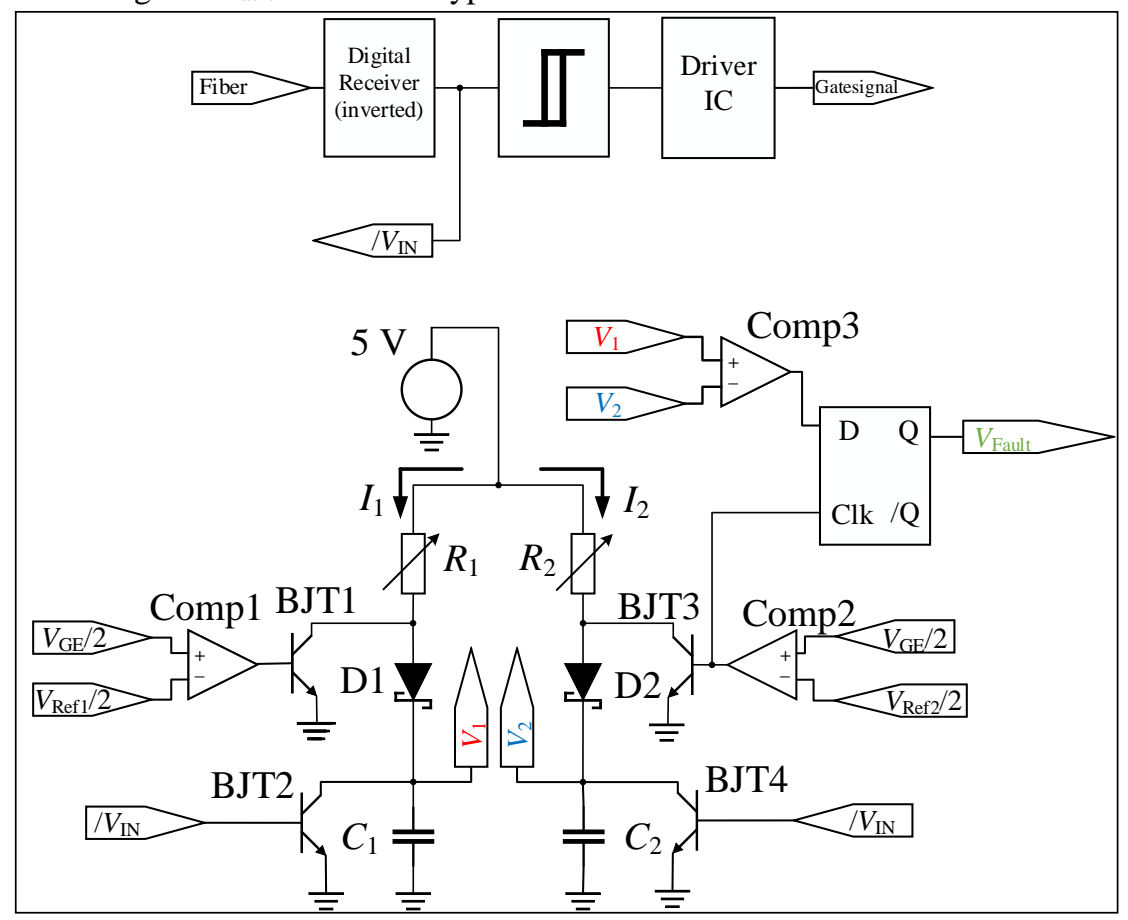

Fig. 1: Gate-signal-path (top) and detection circuit (bottom).

Under normal turn-on conditions, the relation (1) is applied due to a longer charging time $t_{2}$ of $V_{2}$, see Fig. 2:

$$
V_{1}<\left.V_{2}\right|_{t}=t_{2}
$$

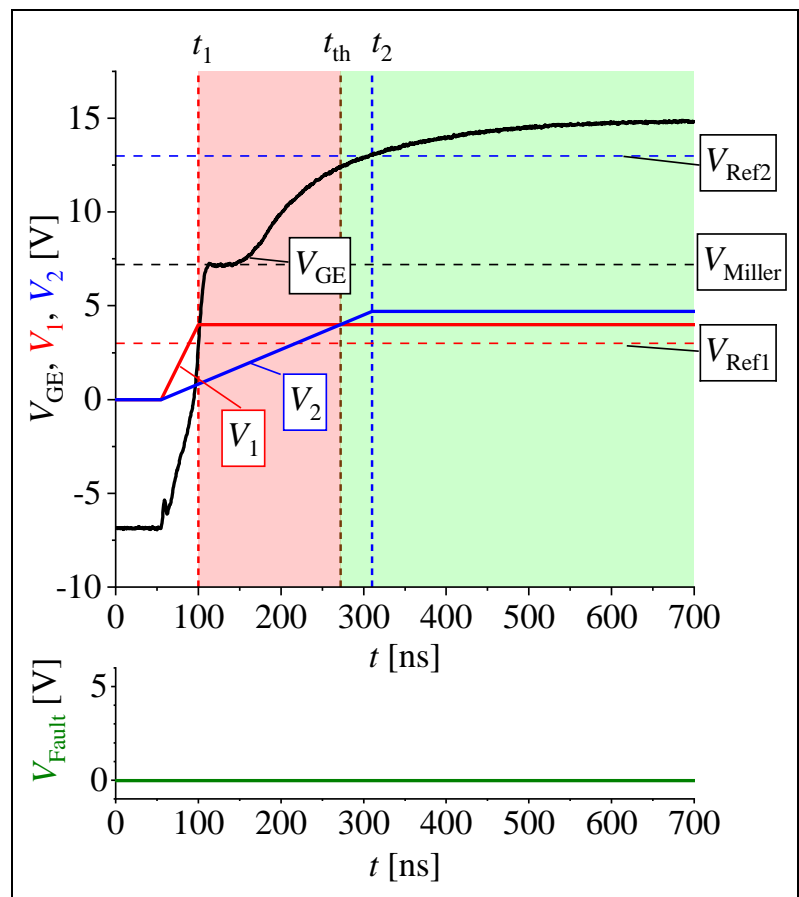

Fig. 2: Working principle with idealistic courses for $V_{1}, V_{2}$ (top), and $V_{\text {Fault }}$ (bottom) under normal switching operation.

On the contrary, under SC conditions $V_{2}$ does not have enough time to reach the value of $V_{1}$, because the rise of $V_{\mathrm{GE}}$ is faster without the Miller-plateau.
Therefore, (2) holds, see Fig. 3. This results in a fault signal $V_{\text {Fault }}$ when the gate voltage reaches $V_{\text {Ref2: }}$ :

$$
V_{1}>\left.V_{2}\right|_{t}=t_{2}
$$

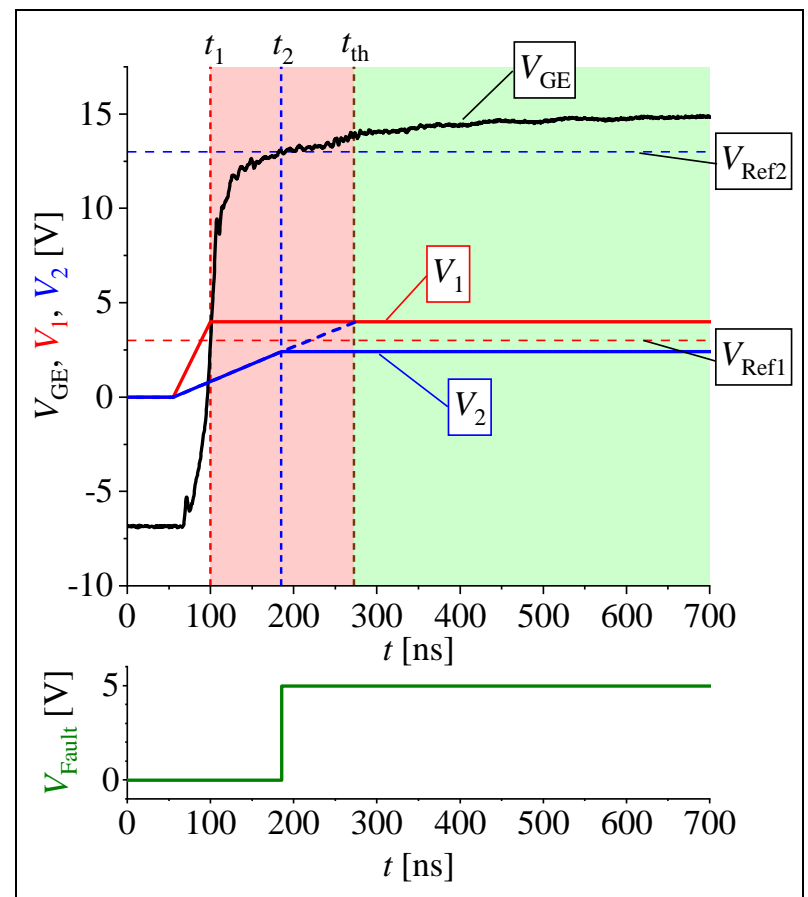

Fig. 3: Working principle with idealistic courses for $V_{1}, V_{2}$ (top), and $V_{\text {Fault }}$ (bottom) under SC I turn-on conditions.

The threshold time $t_{\text {th }}$ is the time when $V_{2}$ exceeds $V_{1}$. Since $t_{\text {th }}$ is dependent on the rise time $t_{1}$ of $V_{1}, t_{\text {th }}$ should automatically be adjusted to a certain value according to the slope of the $V_{\mathrm{GE}}$-course during turn-on. This potentially enables a self-adaption which will be discussed in a later section. 
MEASUREMENT SETUP

To prove the detection method's working principle, application-conform measurements representing normal and SC I turn-on transitions are performed. The load circuit is depicted in Fig. 4. The low-side switch being protected is subjected to a normal turn-on process during the double pulse test. In this case, the high-side consists of a load inductivity $L_{\text {Load }}$ and an antiparallel freewheeling diode FWD. For the SC I test, the high-side is shorted before the turn on. The gate voltage is reduced to half by an ohmic voltage divider consisting of two resistors $R_{\mathrm{div}}$, to prevent the comparator input voltage from exceeding the allowable range.

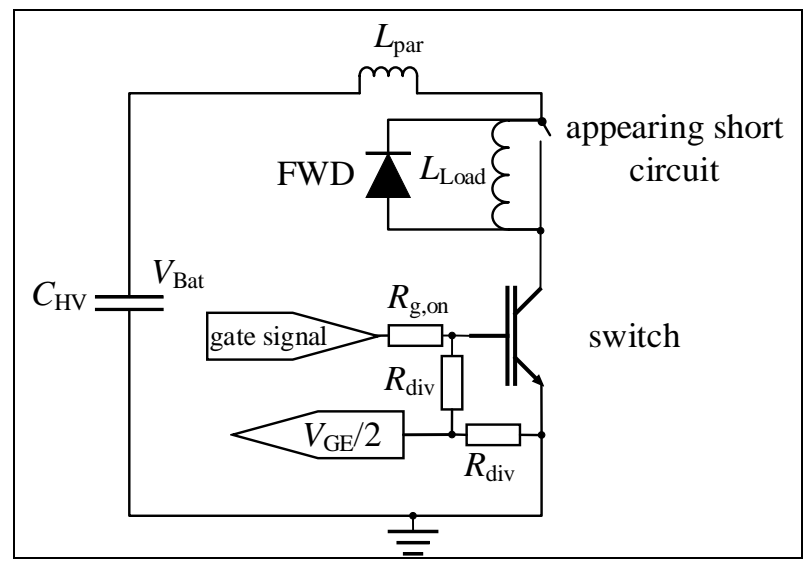

Fig. 4: Load circuit and parts of the gate signal loop for double pulse and SC I test.

The investigations have been carried out with IGBTs in TO-247-3 and EconoPACK-housing from Infineon. In Fig. 5, the proposed circuitry and the module are shown. The PCB on the lower left part of Fig. 5 represents the driver for the switch and its detection circuitry. It was used in this paper for testing IGBTs in both packages as mentioned before.

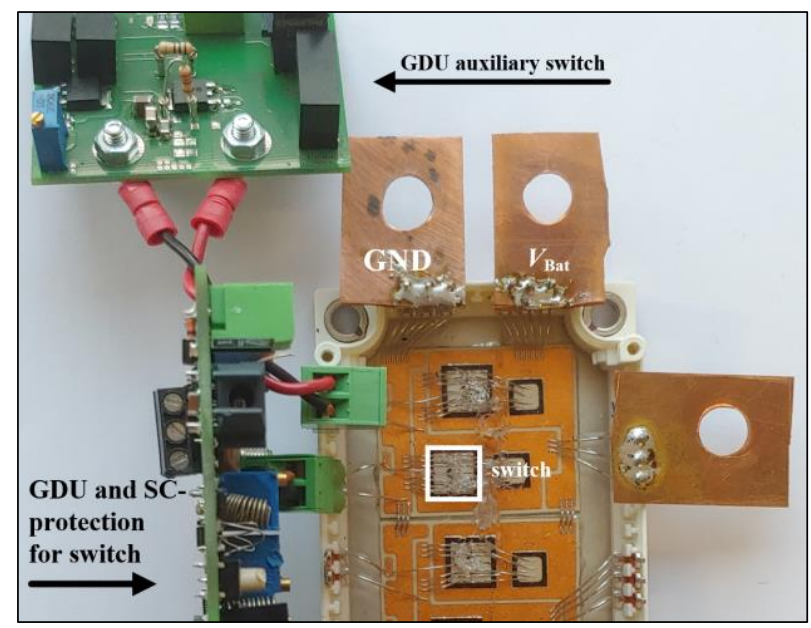

Fig. 5: Connection between EconoPACK-module with Gate Drive Unit (GDU) and detection circuit (bottom left).

\section{MEASUREMENTS FOR GENERAL VALIDATION}

In this section, the general working principle and detection accuracy of the method is demonstrated and proved. The following measurements have been generated using an IGBT-module FS75R17KE3 from Infineon in EconoPACK-housing under applicationcompliant conditions with reference voltages set to $V_{\text {Ref1 }}=3.8 \mathrm{~V}$ and $V_{\text {Ref2 }}=13.8 \mathrm{~V}$.

For the non-fault case (1) see Fig. 6. There is no fault signal given.

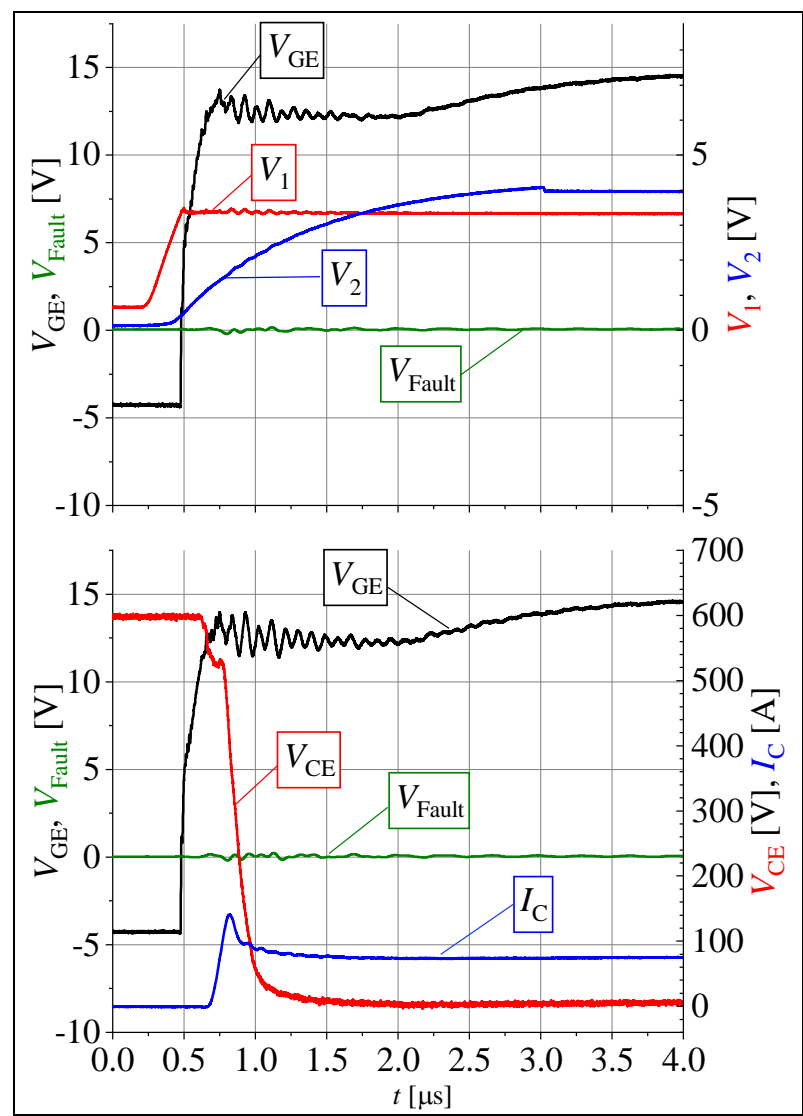

Fig. 6: Courses of detection circuit $V_{1}, V_{2}$ (top), load circuit $V_{\mathrm{CE}}$, $I_{\mathrm{C}}$ (bottom), $V_{\mathrm{GE}}$, and $V_{\text {Fault }}$ during normal turn-on process. $V_{\text {Bat }}=600 \mathrm{~V}, \quad I_{\mathrm{C}}=75 \mathrm{~A}, \quad R_{\mathrm{g}, \text { on }}=6.4 \Omega, \quad L_{\mathrm{Load}}=500 \mu \mathrm{H}$, $L_{\mathrm{par}}=70 \mathrm{nH}$; EconoPACK-module.

For all the partial figures showing measurements, $V_{\mathrm{GE}}$ is always measured and depicted to demonstrate its relation to $V_{1}$ and $V_{2}$ or $V_{\mathrm{CE}}$ and $I_{\mathrm{C}}$. $V_{\text {Fault }}$ is always shown to prove its validity for each measurement.

At the beginning of the Miller-phase LC-oscillations are visible in the $V_{\mathrm{GE}}$ signal excited by the $\mathrm{d} i_{\mathrm{C}} / \mathrm{d} t$ during the current rise and Reverse Recovery event of FWD. Regarding the stray inductance in the gate-loop, the connection between the GDU-detection board and the module is not realized ideally, as can be seen in Fig. 5.

However, this does not affect the functionality of the detection circuit concerning the correct classification of a normal turn-on event. Nevertheless, an optimized gate loop design could mitigate this issue. 
A successfully detected SC measurement under the same conditions as before is shown in Fig. 7. After turning on for around $300 \mathrm{~ns}$, a short circuit is detected under given measurement conditions.

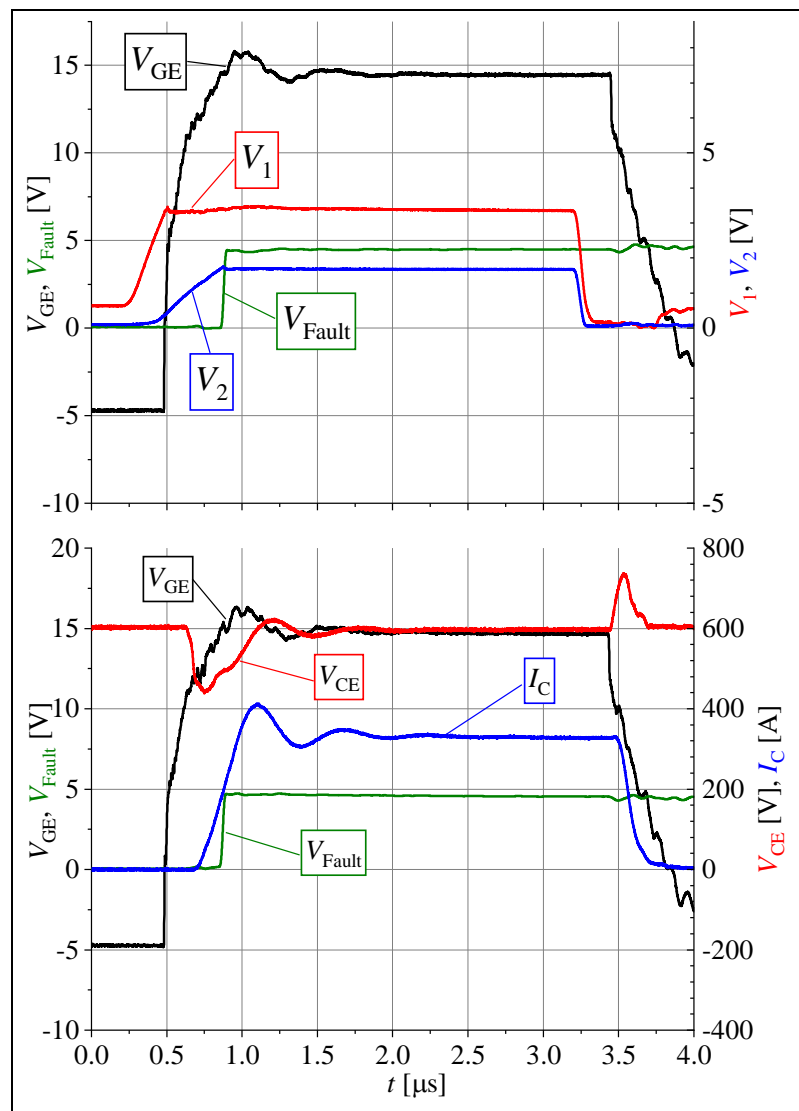

Fig. 7. Courses of $V_{1}, V_{2}$ of the detection circuit (top), $V_{\mathrm{CE}}, I_{\mathrm{C}}$ of the load circuit (bottom), and $V_{\mathrm{GE}}$ and $V_{\text {Fault }}$ during SC I. $V_{\text {Bat }}=600 \mathrm{~V}, \quad I_{\mathrm{C}}=75 \mathrm{~A}, \quad R_{\mathrm{g}, \text { on }}=6.4 \Omega, \quad L_{\text {Load }}=500 \mu \mathrm{H}$, $L_{\mathrm{par}}=70 \mathrm{nH}$; EconoPACK-module.

The first measurements shown in Fig. 6 and Fig. 7 demonstrate that the proposed detection method can successfully distinguish between a normal and a failure turn-on. The detection offers a valid fault signal even before the static value of the positive gate voltage and short circuit current is attained. The detection mechanism can also be used within a 2-level turn-on procedure, where the full positive gate voltage is only released when no fault has been detected in the first turn-on step.

\section{INVESTIGATION ON PACKAGES WITH AND WITHOUT A SENSE PIN}

The evaluation of the behavior of the short circuit detection has not only been carried out on modules in EconoPACK-housing but also on devices in TO-247-3 package. Fig. 8 shows the behavior during a normal turnon process using an IKW20N60T IGBT from Infineon under application relevant conditions. It is visible that, similarly to the EconoPACK-operation, oscillations occur from the appearance of the $\mathrm{d} i_{\mathrm{C}} / \mathrm{d} t$ at turn on. Furthermore, the measured gate voltage $V_{\mathrm{GE}}$ is subjected to a transient increase at the beginning of the Millerphase between 300 and $350 \mathrm{~ns}$.

Although the current slope $\mathrm{d} i_{\mathrm{C}} / \mathrm{d} t$ for the TO-package is lower compared with the module, a more pronounced $V_{\mathrm{GE}}$ overshoot during normal turn-on can still be observed.

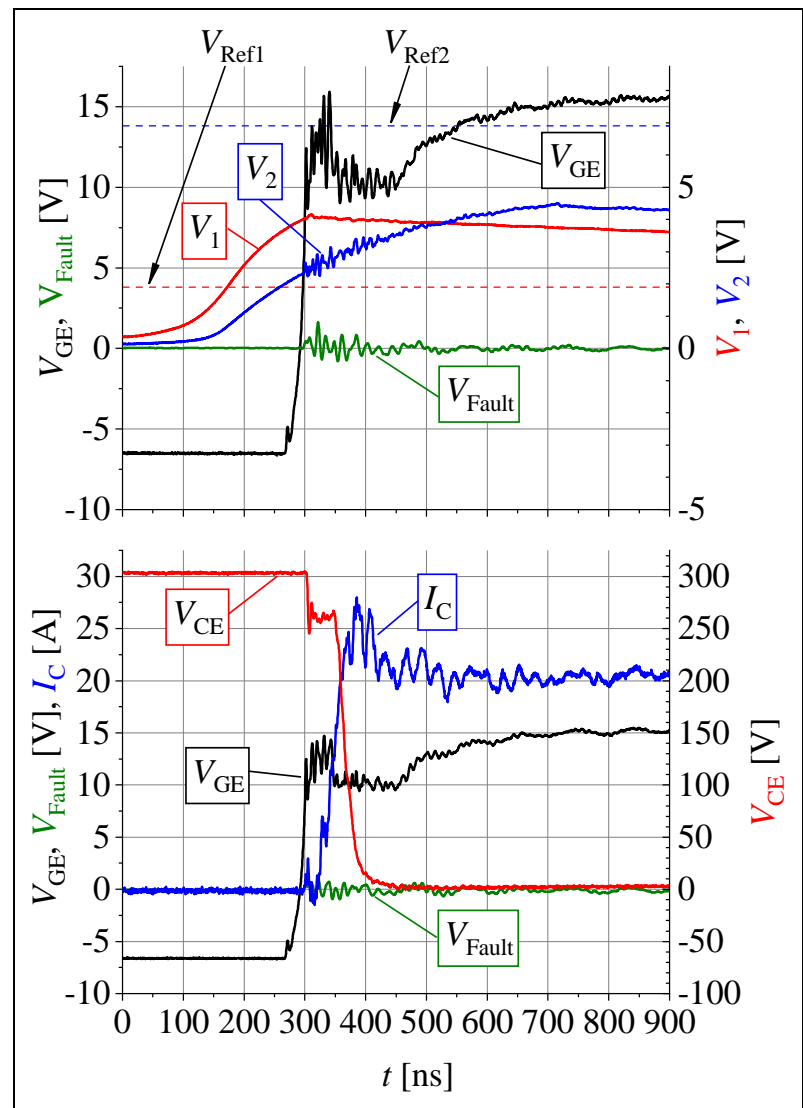

Fig. 8: Courses of $V_{1}, V_{2}$ of the detection circuit (top), $V_{\mathrm{CE}}, I_{\mathrm{C}}$ of the load circuit (bottom), and $V_{\mathrm{GE}}, V_{\text {Fault }}$ during normal turnon process. $V_{\mathrm{Bat}}=300 \mathrm{~V}, \quad I_{\mathrm{C}}=20 \mathrm{~A}, \quad R_{\mathrm{g}, \mathrm{on}}=6.2 \Omega$, $L_{\text {Load }}=500 \mu \mathrm{H}, L_{\mathrm{par}}=80 \mathrm{nH}$; TO-247-3-package.

The reason for this phenomenon can be found in the different structures of the two packages. Fig. 9 shows the gate loop of the module and how it is affected by a positive load current transient $\mathrm{d} i_{\mathrm{C}} / \mathrm{d} t$.

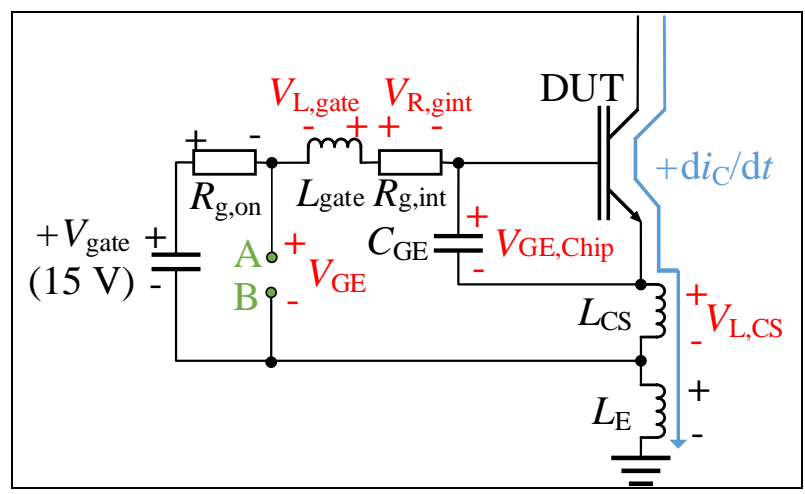

Fig. 9: Structure of the gate loop for a package or module offering a Kelvin sense contact.

The measured gate voltage $V_{\mathrm{GE}}$, which also constitutes the voltage signal being monitored by the detection circuit before division, is picked off at terminals A and B. 
During the occurrence of a collector current slope, $V_{\mathrm{GE}}$ does not correspond to the real chip-internal gate voltage $V_{\mathrm{GE}, \text { chip. }}$ Across the stray inductance in the gate loop $L_{\text {gate }}$ and the common-source inductance $L_{\mathrm{CS}}$ affected by the gate current slope and the collector current slope respectively, additional voltages are induced:

$$
V_{\mathrm{GE}}=V_{\mathrm{R}, \text { gint }}+V_{\mathrm{GE}, \text { chip }}+V_{\mathrm{L}, \mathrm{CS}}-V_{\mathrm{L}, \text { gate }} \text {. }
$$

As

$$
V_{\mathrm{L}, \mathrm{CS}}+V_{\mathrm{R}, \text { gint }}>V_{\mathrm{L}, \text { gate }}
$$

applies, (5) holds.

$$
V_{\mathrm{GE}}>V_{\mathrm{GE}, \text { chip }}
$$

In contrast to the EconoPACK-module, a 3-pin TOpackage is not provided with a separate Kelvin sense contact for the gate driver. For the TO-package, $L_{\mathrm{E}}$ in Fig. 9 is nonexistent, but there is a large $L_{\mathrm{CS}}$. Hence, this measurement error produced by $L_{\mathrm{CS}}$ for a 3-pin TOpackage becomes more pronounced compared to the used module offering a separate Kelvin sense connection.

Furthermore, the so-called self turn-on effect [11] can lead to a transient increase of $V_{\mathrm{GE}}$ additionally for both package concepts.

In the measurements shown in Fig. 8, $V_{\mathrm{GE}}$ exceeds the second reference $V_{\text {Ref2 }}$ before $t_{\mathrm{th}}$, which normally would lead to a stopping of the charging process of $C_{2}$ and the end of the rise of $V_{2}$ accordingly, resulting in the output of a failure signal $V_{\text {Fault }}$. Since the ohmic voltage divider consisting of the two resistors $R_{\text {div }}$, dimensioned with each $2.7 \mathrm{k} \Omega$, is not frequency compensated, $V_{\mathrm{GE}} / 2$ monitored by the detection logic is delayed to the original course of $V_{\mathrm{GE}}$. To ensure fast and precise monitoring of the gate voltage, which this detection approach is aiming at, such a delay is generally considered as a disadvantage regarding the accuracy, especially for fast transitions. However, in the stated example this lack of precision turns into an advantage as it offers a certain level of robustness against the described transient gate voltage overshoot, oscillations, and noise. This results in a correctly detected normal turn-on process for the case depicted by Fig. 8 . Nevertheless, when decreasing $R_{\text {div }}$ to $1 \mathrm{k} \Omega$, the divider proves fast enough to be sensitive to the disturbance resulting in the output of a fault signal constituting a wrongly detected SC I turn on (false positive). Due to this issue, a precise and reliable operation of the detection circuit cannot be ensured and proven under all conditions when applying this detection method to a package missing a Kelvin sense emitter. Instead, the usage of a device with a Kelvin sense emitter is required and recommended.

\section{VARIATION OF TURN-ON RESISTANCE AND SELF-ADAPTION}

Apart from the fast detection speed, the proposed method's capability to dynamically self-adapt should also be given. Specifically, a change of the $V_{\mathrm{GE}}$ slope without any readjustment via $R_{1}$ or $R_{2}$ in Fig. 1 or the reference voltages is highlighted. A change of the switching speed may occur due to a change of the gate turn-on resistor or the input capacitance. The functionality of this $V_{\mathrm{GE}}$-based detection method under an expanded condition set and its claimed self-adaption feature under different $V_{\mathrm{GE}}$ slopes shall be investigated in this section. For this purpose an evaluation under a variation of the $R_{\mathrm{g}, \mathrm{on}}$-values is performed.

\section{Functional capability under a variation of $\boldsymbol{R}_{\mathrm{g}, \mathrm{on}}$}

As the differences of the gate voltage courses get smaller with an increase in switching speed, distinguishing between normal and failure turn-on processes is considered to be more critical for low $R_{\mathrm{g}, \mathrm{on}}$-values.

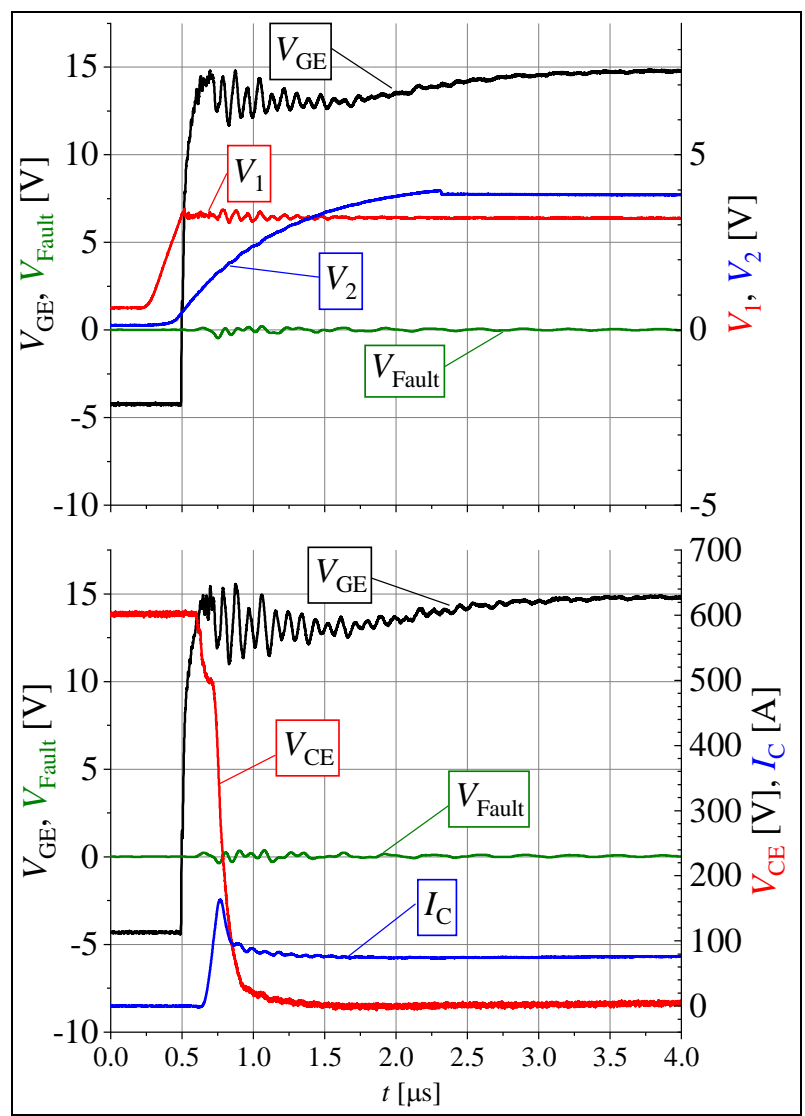

Fig. 10: Courses of detection circuit $V_{1}, V_{2}$ (top), load circuit $V_{\mathrm{CE}}, I_{\mathrm{C}}$ (bottom), $V_{\mathrm{GE}}, V_{\text {Fault }}$ during normal turn-on process. $V_{\text {Bat }}=600 \mathrm{~V}, \quad I_{\mathrm{C}}=75 \mathrm{~A}, \quad R_{\mathrm{g}, \text { on }}=1.7 \Omega, \quad L_{\text {Load }}=500 \mu \mathrm{H}$, $L_{\mathrm{par}}=70 \mathrm{nH}$; EconoPACK-module.

According to the aforementioned results shown in Fig. 6 and Fig. 7, where a datasheet- $R_{\mathrm{g}, \text { on }}$ of $6.4 \Omega$ is used, measurements with an $\mathrm{R}_{\mathrm{g}, \mathrm{on}}$ of $1.7 \Omega$ and $3.5 \Omega$, respectively have been carried out. For both values, the separation of normal and failure cases is working reliably. 
Fig. 10 and Fig. 11 show the measurement results for $R_{\mathrm{g}, \text { on }}=1,7 \Omega$ representatively.

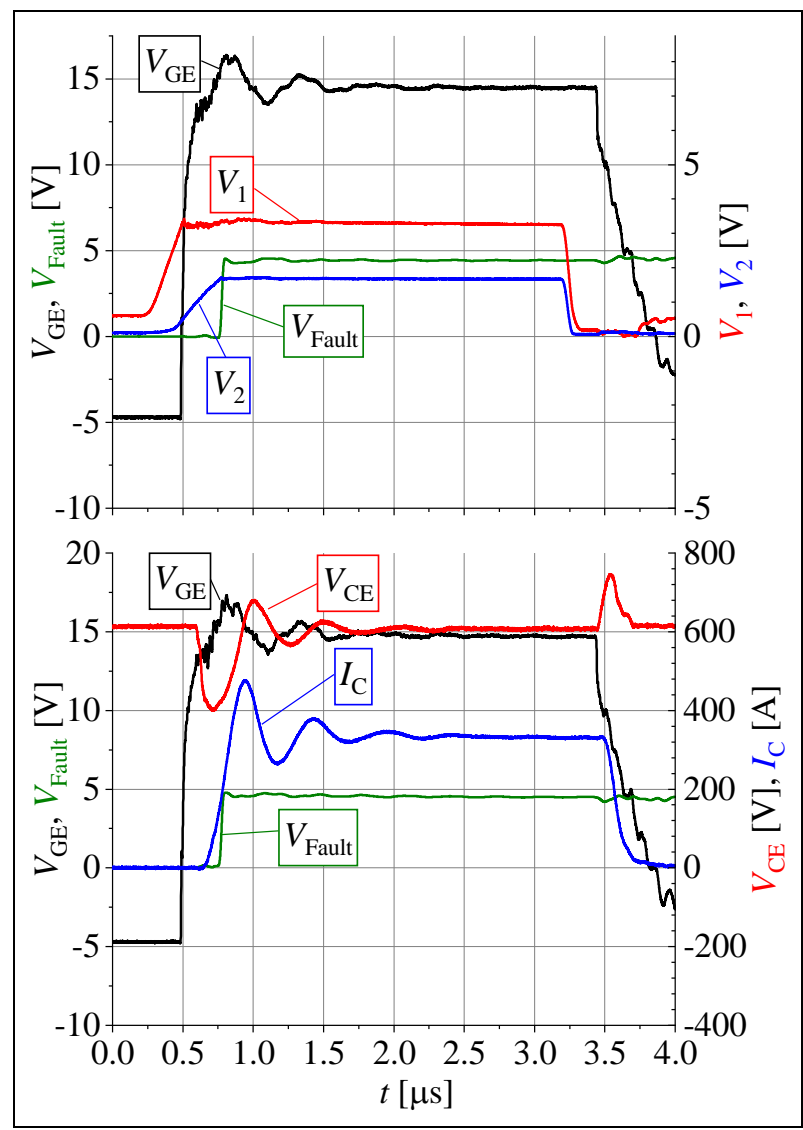

Fig. 11: Courses of $V_{1}, V_{2}$ of the detection circuit (top), $V_{\mathrm{CE}}, I_{\mathrm{C}}$ of the load circuit (bottom) and $V_{\mathrm{GE}}, V_{\text {Fault }}$ during SC I. $V_{\text {Bat }}=600 \mathrm{~V}, \quad I_{\mathrm{C}}=75 \mathrm{~A}, \quad R_{\mathrm{g}, \text { on }}=1.7 \Omega, \quad L_{\mathrm{Load}}=500 \mu \mathrm{H}$, $L_{\mathrm{par}}=70 \mathrm{nH}$; EconoPACK-module.

\section{Investigation on self-adaptive behavior}

Conventional gate voltage-based SC I detections rely on attaining one reference voltage level within a certain time to detect a potential short. A big advantage of the proposed detection method is claimed to be the utilization of two reference voltages [5]. In theory, this allows the detection circuitry to dynamically self-adapt to different $V_{\mathrm{GE}}$ slopes, as the time $t_{\text {th }}$ will automatically shift to certain values when $t_{1}$ is changed due to flatter or steeper $V_{\mathrm{GE}}$ slopes.

For a conventional gate voltage-based SC I detection the top of Fig. 12 depicts the $V_{\mathrm{GE}}$-courses of normal and failure turn-on processes for a variety of $V_{\mathrm{GE}}$ slopes schematically. $t_{\mathrm{th}}$ is fixed to a constant value, represented by the constant $V_{1} . V_{2}$ is, similarly to the method discussed in this paper, used to assess the turn-on speed and to distinguish between a normal and a failure turn-on (see Fig. 12 bottom). As a result, the conventional approach would classify both a fast normal turn-on (Fig. 12: steep slope, normal), as well as a slow failure turn-on (Fig. 12: flat slope, SC I) incorrectly. Only the case for medium slopes is assessed correctly, as $t_{\text {th }}$ fits to the proper separation of normal and SC I turn on.

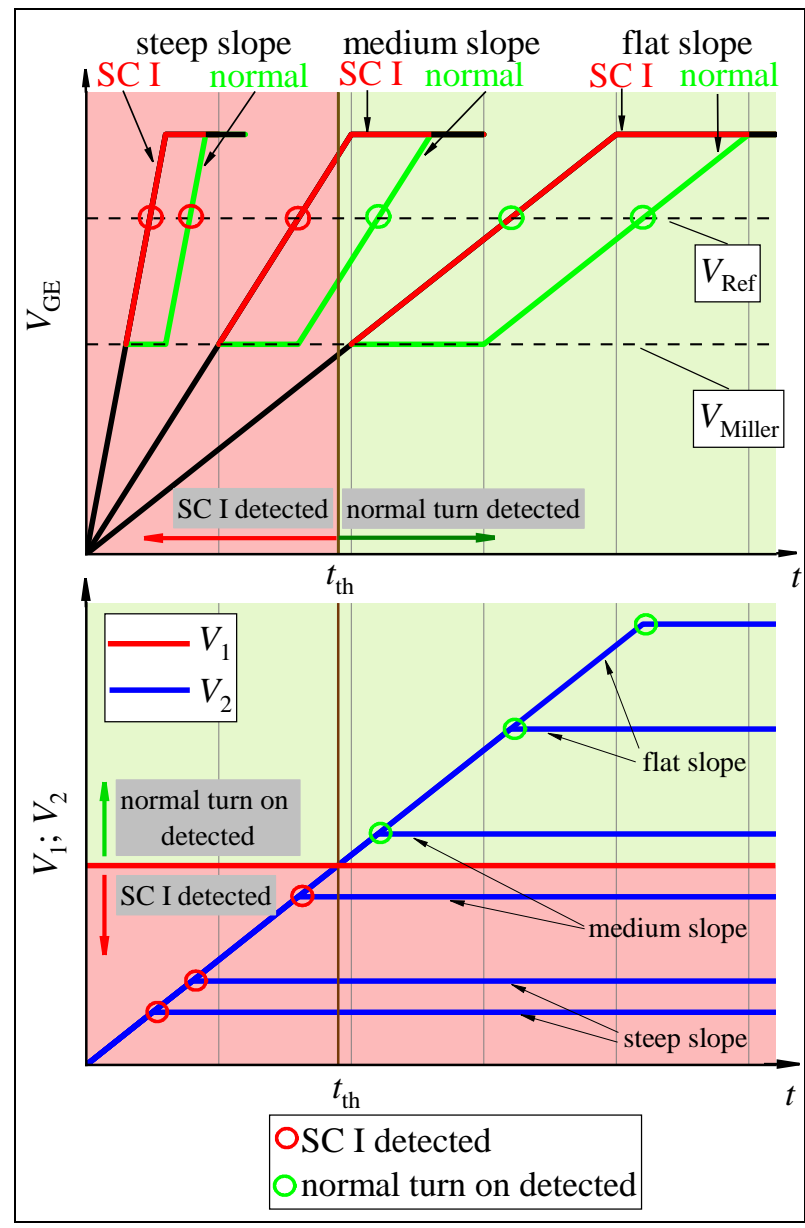

Fig. 12: Simplified schematic courses of $V_{\mathrm{GE}}$ for three different slopes under normal and low inductive SC I turn-on conditions for conventional gate voltage based method (top). Courses of the detection circuitry with only one voltage reference ( $V_{\text {Ref, }}$ top, represented by $V_{1}$, bottom). To provide comparability with the approach presented in this paper, $V_{1}$ is set to a constant value, representing the fixed threshold time $t_{\text {th }}$ (see also [5]).

In contrast, the proposed principle relying on two voltage reference levels should categorize all switching events correctly. This can be achieved because $t_{\text {th }}$ is correlated to the variable value of $t_{1}$. Therefore, $t_{1}$, describing the time within which $V_{\text {Ref1 }}$ is attained, acts as a determining factor and is used to adjust $t_{\text {th }}$ and set it to a certain value according to the switching speed being present in the

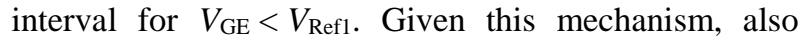
schematically depicted in Fig. 13 for the steep and flat slope case, a self-adaption to different switching speeds shall be enabled without the necessity to readjust the circuitry. The appropriate schematic courses of $V_{1}$ and $V_{2}$ are drawn in the lower part of Fig. 13. 


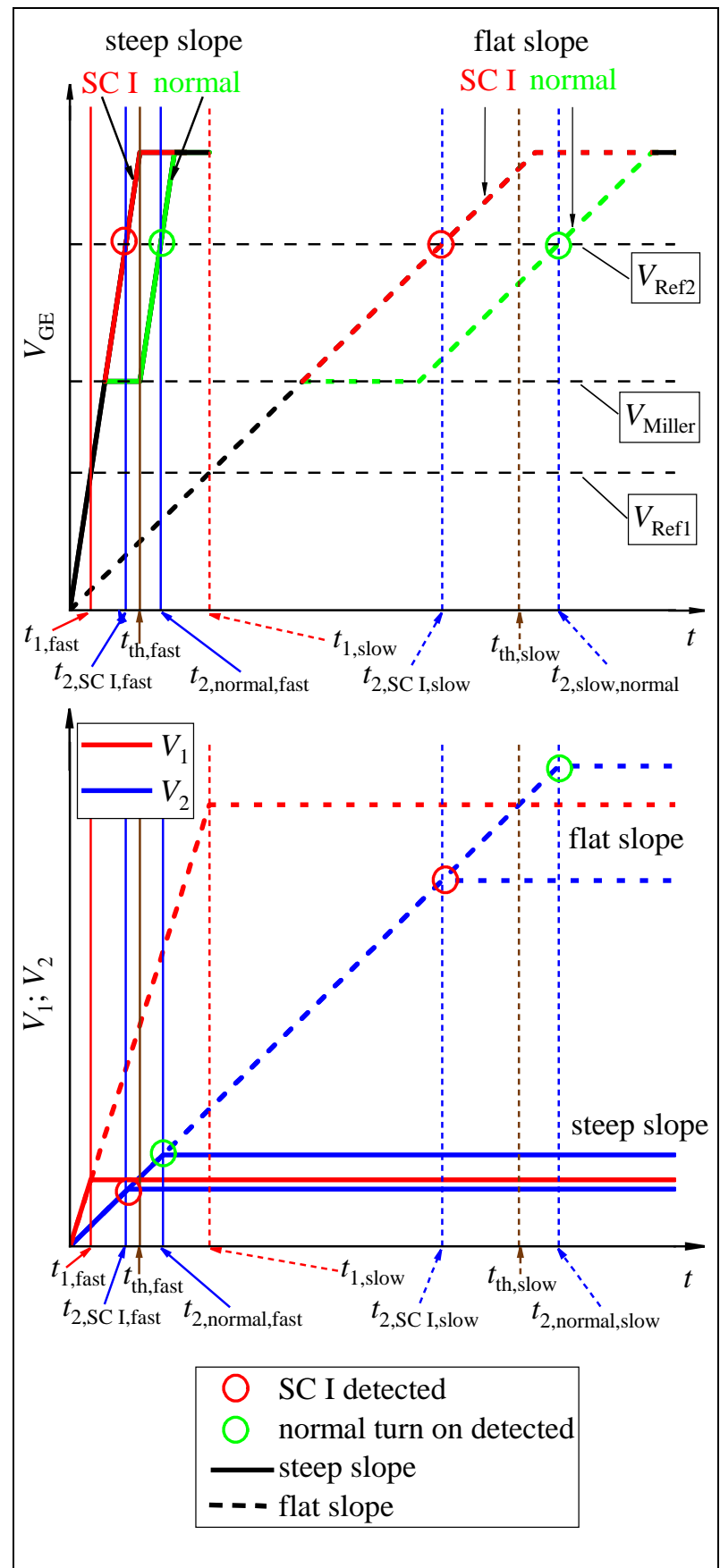

Fig. 13: Simplified schematic courses of $V_{\mathrm{GE}}$ for the steep and flat slope cases of Fig. 12 under normal turn-on and SC I conditions (top). Courses of the proposed detection circuitry using two voltage reference levels (bottom). $t_{\text {th }}$ is set dynamically according to $t_{1}$, which adapts to the slope of $V_{\mathrm{GE}}$ (see also [5]).

Regarding the measurements shown in this paper for the EconoPACK-housing, the correct classification of the switching events for different $R_{\mathrm{g}, \text { on }}$-values between $1.7 \Omega$ and $6.4 \Omega$, shown in Fig. 6, Fig. 7, Fig. 10 and Fig. 11, could be obtained without a readjustment of the detection circuitry. Nevertheless, this achievement does not rely on dynamic self-adapting behavior. Fig. 14 displays the comparison of the $V_{\mathrm{GE}}$-courses for the fastest normal turn-on at $R_{\mathrm{g}, \mathrm{on}}=1.7 \Omega$ and the slowest SC I turn-on at $R_{\mathrm{g}, \mathrm{on}}=6.4 \Omega$.

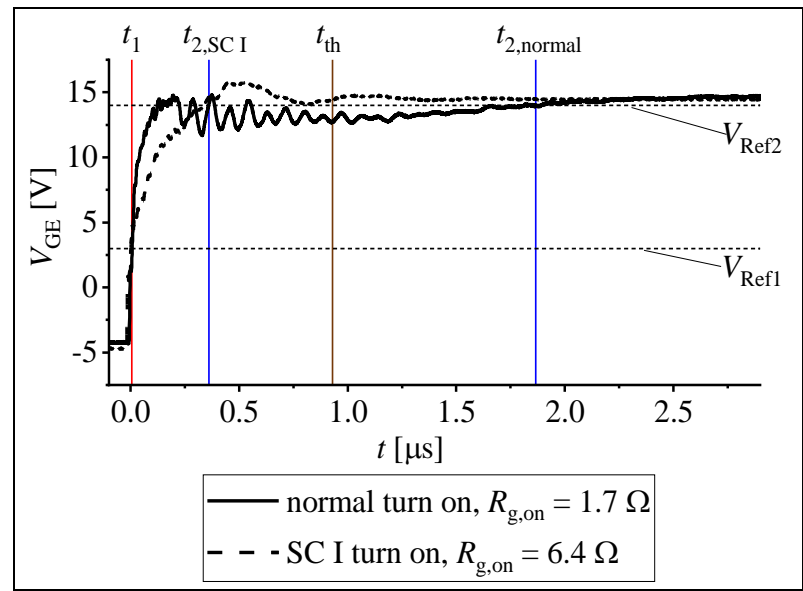

Fig. 14: $V_{\mathrm{GE}}$ for the fastest normal turn on at $R_{\mathrm{g}, \mathrm{on}}=1.7 \Omega$ and the slowest SC I turn on at $R_{\mathrm{g}, \text { on }}=6.4 \Omega$.

The slight exceedance of $V_{\text {Ref2 }}$ at the beginning of the Miller-phase is not taken into consideration by the detection circuitry because of the already explained smoothing behavior of the ohmic voltage divider. Despite the smaller $R_{\mathrm{g}, \text { on }}=1.7 \Omega$, the normal turn-on process is still slower than the SC I turn on at higher $R_{\mathrm{g}, \text { on }}(6.4 \Omega)$ regarding the surpassing of the second reference $V_{\text {Ref2 }}$. The correct categorization of both the cases shown in Fig. 14 without any readjustment is based on this fact instead of a dynamic self-adaption.

In contrast to the theoretic considerations of Fig. 12 (top) and Fig. 13 (top), the crossing of the first reference $V_{\text {Refl }}$ does not change significantly with a variation of $R_{\mathrm{g}, \text { on }}$ within a certain range. As a result, a dynamic selfadaption would not happen in this case. The weak dependence of $t_{1}$ on $R_{\mathrm{g}, \text { on }}$ is also due to the relatively high internal gate resistance $R_{\mathrm{g}, \text { int }}=8.5 \Omega$ present in the DUT, which attenuates changes of the external gate resistance. The self-adaptive behavior can only be proven, when the SC I turn-on is slower than the normal turn-on across the entire $V_{\mathrm{GE}}$-course (see Fig. 13). In this case, a conventional gate voltage-based detection method would fail (see Fig. 12). Furthermore, a self-adaption could potentially only take place if the surpassing of $V_{\text {Refl }}$ occurred at considerably different times $t_{1}$ for both cases so that an adjustment of $t_{\text {th }}$ could take place.

\section{CONCLUSION}

A discrete gate voltage-based SC I detection introduced in [5] has been presented and tested. The results show that the proposed method can detect a low inductive SC type I failure in a very fast and reliable way. The failure will be detected when $V_{\mathrm{GE}}$ attains the second voltage reference level. For the used 75 A EconoPACK-module, the SC I was detected $270 \mathrm{~ns}$ and $300 \mathrm{~ns}$ after turn-on for external gate turn-on resistances of $1.7 \Omega$ and $6.4 \Omega$, respectively. Subsequently, for different gate resistors and input capacitances, the detection speed varies.

Furthermore, the reliability of the method highly depends on the package form of the DUT. 
The reliability and functionality are applied for a package offering a sense emitter contact with a low $L_{\mathrm{CS}}$ design. The packages, which are not provided with a Kelvin sense, such as 3-pin TO-247 devices, as tested in this paper, are not recommended. Special attention should be paid to an operation at high load currents or turn-on transitions with low gate turn-on resistances since the voltage level of the Miller plateau depends on the level of load current and IRRM. This aspect can affect the detection method's functionality when an overload current or a high IRRM is observed.

The expected self-adaptation of the detection for a varied $V_{\mathrm{GE}}$ slope highly depends on the DUT's chip design, such as internal gate resistance and input capacitance $C_{\text {iss }}$ (current rating).

However, the detection method demonstrated in this paper appears to be suitable also for bigger modules such as IHM (IGBT High Power Module), since the internal gate resistance is usually small and the common-source inductance $L_{\mathrm{CS}}$ is significantly optimized. Moreover, the high $C_{\text {iss, }}$ due to a large number of chips in parallel, enables a wide detection time window for the varied $V_{\mathrm{GE}}$ slope. Finally, in comparison to the conventional $V_{\mathrm{CE}^{-}}$ monitoring, the gate driver board is not exposed to high voltage, which provides more safety for the control unit and the driver itself.

\section{REFERENCES}

[1] Hain, S. and Bakran, M.-M.: New Ultra Fast Short Detection Method Without Using the Desaturation Process of the Power Semiconductor, Proc. PCIM Europe 2016 (Nuremberg, 2016), 720-727

[2] Hain, S. and Bakran, M.-M.: Integrating the new 2D Short Circuit Detection Method into a Power Module with a Power Supply fed by the Gate Voltage, IEEE 2nd Annual Southern Power Electronics Conference (SPEC) (Auckland, 2016), 1-6

[3] Rüedi, H. and Köhli, P.: Driver Solutions for High-voltage IGBTs, Proc. PCIM Europe 2002 (Nuremberg, 2002), 1-11

[4] Huang, F. and Flett, F.: IGBT Fault Protection Based on di/dt Feedback Control, Proc. 2007 IEEE Power Electronics Specialists Conference (Orlando, 2007), 1478-1484

[5] Miyazaki, K., Omura, I., Takamiya, M. and Sakurai, T.: 20-ns Short-Circuit Detection Scheme with High VariationTolerance based on Analog Delay Multiplier Circuit for Advanced IGBTs, Proc. IEEE 2nd Annual Southern Power Electronics Conference (Auckland 2016), 1-4

[6] Fuhrmann, J., Hammes, D., Muenster, P., Lexow D. and Eckel, H.-G.: Short-circuit detection based on gate-emitter voltage of high-voltage IGBTs, Proc. $19^{\text {th }}$ European Conference on Power Electronics and Applications (Warsaw, 2017), 1-9
[7] Hain, S. and Bakran, M.-M.: The suitability and challenges of the new 2D-short circuit detection method for protecting a high performance IGBT with a low $\mathrm{V}_{\text {ce,sat }}$ value, Proc. $18^{\text {th }}$ European Conference on Power Electronics and Applications (Karlsruhe, 2016), 1-10

[8] da Cunha, J., Fuhrmann, J., Lexow, D., Hammes, D. and Eckel, H.-G.: A new Combined $V_{\mathrm{GE}}$ and $\mathrm{V}_{\mathrm{CE}}$ Based ShortCircuit Detection for High-IC,desat HV-IGBTs, Proc. PCIM Europe 2018 (Nuremberg, 2018), 825-832

[9] Lee, J.-B. and Hyun, D.-S. Gate Voltage Pattern Analyze for Short-Circuit Protection in IGBT Inverters, Proc. 2007 IEEE Power Electronics Specialists Conference (Orlando, 2007), 1913-1917

[10] Park, B.-G., Lee, J.-B. and Hyun, D.-S.: A Novel ShortCircuit Detecting Scheme Using Turn-on Switching Characteristic of IGBT, Proc. 2008 IEEE Industry Applications Society Annual Meeting (Edmonton, 2008), 1-5

[11] Münster, P., Tran, Q. T., Lexow, D. and Eckel, H.-G.: Current imbalance affected by self turn-ON during turn-ON of paralleled HV-IGBTs, Proc. 18th European Conference on Power Electronics and Applications (Karlsruhe, 2016), 1-9

Addresses of the authors

Clemens Herrmann, Reichenhainer Str. 70, Chemnitz, clemens.herrmann@etit.tu-chemnitz.de Xing Liu, Reichenhainer Str. 70, Chemnitz Josef Lutz, Reichenhainer Str. 70, Chemnitz Thomas Basler, Reichenhainer Str. 70, Chemnitz 